\title{
What do anaesthetists think of sedation by GDPs?
}

\author{
A survey of the opinions of consultant anaesthetists in Scotland of sedation carried out by dentists \\ J. Shearer, K. E. Wilson and N. M. Girdler Br Dent J 2003; 196: 93-98
}

\section{Objectives}

To elicit the attitudes and opinions of consultant anaesthetists working in Scotland, with regard to conscious sedation carried out by dental practitioners.

\section{Method}

A questionnaire was designed to gauge opinion of consultant anaesthetists in Scotland on the practice of conscious sedation by dentists. The questionnaire was sent to 353 consultant anaesthetists working in 49 hospitals within the 15 health boards in Scotland.

\section{Results}

Of the 366 questionnaires sent, 249 were returned of which 235 were valid. This gave a response rate of $64 \%$. In general, those questioned felt that the provision of sedation in a hospital setting was more appropriate than in general dental practice. A majority (65\%) thought that it was unrealistic for anaesthetists to provide all sedation for dental treatment, although many (58\%) felt that anaesthetists should take more responsibility in this area. Again, a majority (60\%) agreed that dentists should be trained to use sedation techniques for their patients but a significant number (63\%) disagreed with the practice of operator/sedationist.

\section{Conclusion}

It is of concern to the dental profession that a significant number of anaesthetists do not feel that it is appropriate for dentists to be administering even the most simple methods of sedation. At present there are no clear, recognised guidelines as to the level of formal training required for the practice of conscious sedation by dentists. It is in the interests of the dental profession and the public to ensure that those choosing to practice sedation do so safely by following recognised guidelines in the training and practice of sedation.

\section{IN BRIEF}

- This paper shows the number, length of qualification and involvement in dental work of anaesthetists in Scotland.

- It gives an interesting insight into drugs commonly used in dental sedation.

- Safety aspects of sedation are considered.

- The attitudes of consultant anaesthetists to sedation provided by dentists are varied and interesting.

- Inconsistencies in sedation training are highlighted.

\section{COMMENT}

Dentistry and anaesthesia have been inextricably linked since the first anaesthetic was described in the mid-19th century. Many dentists have excellent working relationships with their anaesthetic colleagues - collaboration, particularly in the field of research, is common and increasing.

The high profile of pain and anxiety control in dentistry, evidenced by the increased literature on the subject and the increased number of courses available throughout the UK, makes this study both timely and fascinating.

The headline news is that much more work needs to be done on education of, and co-operation with, our anaesthetic colleagues. Almost $60 \%$ of those questioned felt dentists should be trained in sedation techniques; $63 \%$ expressed considerable reservation with the concept of operator/sedationist. Quite remarkably, 55 of the anaesthetists thought dentists should not use inhalational sedation with nitrous oxide and oxygen - a technique developed within dentistry and safely practised for many years. Four per cent felt sedation was unnecessary for adult patients in dentistry and there seems to be little knowledge of dental nurse education.

It is very interesting to note that only $12 \%$ of those responding actually provided any intravenous sedation for dentistry, and they therefore, it could be argued, come from a slightly less than informed baseline.

It is now widely accepted amongst those in charge of anaesthesia that sedation is not lite-anaesthesia, and should be taught accordingly. Dentistry is at the forefront of sedation research and education, often in collaboration with our more enlightened anaesthetic colleagues. The Dental Teachers Sedation Group has developed a curriculum and recommended minimum training standards, which although written for undergraduate training can be applied at any level. The question is not whether dentists should be trained or whether sedation should be offered, but how we best provide that training and service. The best system will inevitably be a combined approach with our anaesthetic colleagues. More difficult cases will benefit from their expertise.

This study clearly demonstrates that more work is necessary to not only inform our anaesthetic colleagues what is presently available, but also how much the service is required. There is still much research to be done in the areas of polypharmacy and the use of drugs such as propofol, but it would appear that many anaesthetists throughout Scotland still have a difficulty even with the well-established techniques of conscious sedation used daily in many dental practices. This needs to be addressed.

J. Leitch, Lecturer in Dental Sedation, Glasgow Dental Hospital and School doi:10.1038/sj.bdj.4810899 